\title{
Latency of Wireless Sensor Networks with Uncoordinated Power Saving Mechanisms
}

\author{
Olivier Dousse \\ LCA-I\&C-EPFL \\ $\mathrm{CH}-1015$ Lausanne \\ Switzerland \\ olivier.dousse@epfl.ch
}

\author{
Petteri Mannersalo \\ VTT Technical Research \\ Centre of Finland \\ P.O.Box 1202, 02044 VTT \\ Finland \\ petteri.mannersalo@vtt.fi
}

\author{
Patrick Thiran \\ LCA-I\&C-EPFL \\ $\mathrm{CH}-1015$ Lausanne \\ Switzerland \\ patrick.thiran@epfl.ch
}

\begin{abstract}
We consider a wireless sensor network, where nodes switch between an active (on) and a sleeping (off) mode, to save energy. The basic assumptions are that the on/off schedules are completely uncoordinated and that the sensors are distributed according to a Poisson process and their connectivity ranges are larger or equal to their sensing ranges. Moreover, the durations of active and sleeping periods are such that the number of active nodes at any particular time is so low that the network is always disconnected.

Is it possible to use such a network for time-critical monitoring of an area? Such a scenario requires indeed to have bounds on the latency, which is the delay elapsed between the time at which an incoming event is sensed by some node of the network and the time at which this information is retrieved by the data collecting sink. A positive answer is provided to this question under some simplifying assumptions discussed in the paper. More precisely, we prove that the messages sent by a sensing node reach the sink with a fixed asymptotic speed, which does not depend on the random location of the nodes, but only on the network parameters (node density, connectivity range, duration of active and sleeping periods). The results are obtained rigorously by using an extension of first passage percolation theory.
\end{abstract}

\section{Categories and Subject Descriptors}

G.3 [Probability and Statistics]: Stochastic processes; C.4 [Performance of Systems]: Modeling techniques

\section{General Terms}

Performance, theory

\section{Keywords}

Sensor networks, continuum percolation,first passage percolation

${ }^{*}$ Corresponding author.

Permission to make digital or hard copies of all or part of this work for personal or classroom use is granted without fee provided that copies are not made or distributed for profit or commercial advantage and that copies bear this notice and the full citation on the first page. To copy otherwise, to republish, to post on servers or to redistribute to lists, requires prior specific permission and/or a fee.

MobiHoc'04, May 24-26, 2004, Roppongi, Japan.

Copyright 2004 ACM 1-58113-849-0/04/0005 ...\$5.00.

\section{INTRODUCTION}

Wireless multihop networks raise challenging and fundamental questions on their scalability. The past few years have seen the first answers to some of these questions, under strongly simplifying assumptions on the physical features of the radio channels, mobility patterns, power assignments, etc., which are required to maintain the problem tractable.

In this paper, we consider more specifically wireless sensor networks, made of a large number of nodes with limited processing and communication capabilities. Each node performs some sensing of a particular confined area, and sends the result to a data collecting node (called sink) in a multihop fashion, using other nodes as relays. These networks need to provide at the same time a good coverage of the area to be monitored and a good connectivity of the network. However, compared to general purpose wireless ad hoc networks, where the latter property is the requirement that the network is fully connected, here it can be somewhat relaxed to the less restrictive requirement that the sink be connected (in a multihop fashion) to a set of nodes that span the entire monitored domain. Indeed, the production cost of a sensor is relatively modest, at least compared to the cost of replacing a failed node in some scenarios, where nodes cannot be easily accessed. This fact, combined with the highly variable quality of the wireless channel and the limited battery lifetime of a sensor, makes it more expensive to ensure the full connectivity of the network than to ensure only that the sink is connected to sensors well scattered over the whole monitored area.

The question of having one node (the sink) connected to a large number sensors well dispersed throughout the domain is central to percolation theory, which will prove to be a very useful tool to solve the problems raised in this paper. The percolation probability is the probability that an arbitrary node (in particular, the sink node) belongs to a cluster of infinite size. The main result of percolation theory is that there exists a finite, positive value of the connectivity range, or equivalently of the node spatial density, under which the percolation probability is zero (sub-critical phase) and above which it is non zero (super-critical phase).

If the connectivity can be made less restrictive in sensor networks than in many other ad hoc networks, energy consumption is often a much more critical variable, because of the limited battery that can be put in a sensor as well as of the cost of replacing a node that has failed. Energy is consumed by sensors in their sensing, processing and communicating tasks. Sensing has to be done at a periodicity dictated by the monitored event. The energy it consumes can be reduced if the area covered by a single sensor is small (and therefore if the number of sensors is large enough). Processing 
and communication energy consumption depends on the hardware, but also on the way data is aggregated and medium is accessed. This consumption is the one offering probably the largest potential for reduction. Indeed, nodes spend a considerable amount of energy in listening to their neighbours, and as long as none of these neighbours transmits any data, this energy is simply wasted. Data collection and medium access control (MAC) schemes need therefore to incorporate energy saving as a primary goal, and proposals for such algorithms have recently emerged in the literature, which show indeed that significant energy savings can be achieved. In particular, most proposals for energy saving MAC schemes for sensor networks introduce a sleeping mode for nodes, during which practically no energy is spent $[23,26]$.

Introducing a sleeping mode does however come at some cost. A solution is to use a Time Division Multiple Access (TDMA) scheme, but this requires nodes to synchronize with each other quite tightly, which can be a quite complex task in large networks with random node locations and imperfect (drifting) clocks. Letting the nodes set their wake-up and sleeping times in a decentralized fashion reduces this complexity, but this increases the delay (also called latency) to transfer information between the sink and a distant node. Pushing the decentralization to an extreme where nodes go to sleep independently from each other, which is the solution we adopt in the present paper, eliminates the complexity of having synchronized clusters of nodes, but at the same time raises concerns about an increase of the latency of the network. More importantly, it will not only increase the average latency itself, but it will also increase the variance of this latency. For some applications, such as spatial data collection for statistical purposes, this is certainly acceptable, but not for many others that are much more time-critical. A typical and important example of such a scenario is the use of sensor network for monitoring an area and sending an alarm when an abnormal event is sensed occurs (such as an intrusion, a rapidly changing variable, etc.). Even if some fixed amount of latency can be tolerated, a highly variable latency due to the random position of the nodes, the random radio range, the non-synchronized or even random sleeping and active periods is much more problematic. Is it possible to let nodes go into sleep, without any coordination between their schedules, and yet have rigorous bounds on the latency?

This paper provides a positive answer to this question, and presents the first analytical bounds on the latency of a sensor with random i.i.d. active and sleeping periods. We use dynamic percolation theory to obtain these bounds, which are based on the following assumptions:

- Nodes are randomly scattered on the plane as a homogeneous Poisson process;

- Nodes can send data in one hop to a neighbour within some prescribed, deterministic connectivity range;

- Nodes switch between an active (“on" state) and a sleeping ("off" state) mode independently from each other;

- When a node senses an event, or when it receives data (message) from one of its neighbours, it stays active and broadcasts this message to all its neighbours within direct reach, until it is sure that the message has reached all its neighbours, or the sink, with high probability;

- As long as nodes do not sense any event, or do not receive any data from their neighbors, the duration of their on and off periods are two independent sequences of i.i.d. random variables. Off periods are either constant or exponentially distributed, while on periods are identically distributed but not necessarily exponentially;

- The sensing range is smaller than or equal to the connectivity range. The sensing range is randomly distributed between a minimal non zero value, and a maximum which is equal to the connectivity range.

The two first assumptions mean that we model the sensor network as a Poisson Boolean model. The assumption of circular direct connectivity area is probably the most debatable among all the assumptions made above [11]. In real life, the connectivity range is far from being a circle with fixed radius in many circumstances: it varies a lot, depending on many factors, such as interferences with other nodes, background noise, time-varying channel, hardware defects, directional antennas, etc. A more realistic model is to set a threshold on the signal to noise and interference ratio at the receiver, as in $[11,1]$, which defines a direct connectivity area around a node by contour plots, whose shape is indeed highly variable. We should note however that percolation does hold for models taking interferences into account [7], under some conditions, when it holds for the Poisson Boolean model. Moreover, when nodes have long sleeping periods, interferences from other nodes become less critical. A second remark should be made relative to the high level of directionality in the reception sets, which is not captured by an isotropic model like the Boolean model. Quite interestingly, anisotropy does help to make the network percolate, and hence to increase connectivity. It has been shown [10] that grains with a circular shapes are the most difficult, among all convex grains with the same surface, to have the network percolate. Consequently, although being certainly a crude model of the real connectivity graph of a sensor networks, the Boolean model provides a conservative estimate of the actual connectivity of a sensor network. The only aspect which is not included in the static Boolean model is the time variability of the wireless channel. It can however be included in a dynamic Boolean model, like the blinking Poisson Boolean model we propose in this paper.

The assumptions on the durations of the active and sleeping periods are rather weak, they resume to independence and identical distributions, with off-period being constant (corresponding to a periodic on/off schedule) or exponentially distributed (corresponding to a memoryless sleeping schedule). We do not require that the on periods follow any specific distribution. The assumption that nodes broadcast any data they sense or receive to all their neighbors makes the routing simple and enables to compute analytically the bounds, while staying reasonable if incoming events rarely occur (in an intrusion detection scenario for example). Finally, it is reasonable to assume that the sensing radius of a sensor is smaller than its transmission range. This means that there will be supercritical amount of devices for message transmissions. In order to save batteries, it is natural that only a fraction of sensor are listening the transmission channel.

Since energy saving is central to sensor networks [9], it has received a considerable amount of attention, driving routing algorithms (see e.g. $[3,22]$ ), scheduling (see e.g. $[20,8]$ ), data collection and aggregation (see e.g. [15]) and MAC (see e.g. [22, 27]). Scheduling strategies trading off energy saving and latency are devised in [28], while a Markovian model exploring the performance of a wireless network with on/off periods is described in [4], under the assumption that there is a path from any sensor to the sink. Here we do not look at the scheduling, routing, data aggregation algorithms, but at the latency of the network. Contrary to all these papers, we do not assume that there is a path from any sensor to the sink. On the contrary, we show that even if there is no connectivity 
of all sensors at all times because many of them are sleeping and only a few are active, it is possible to transfer data from any sensor to the sink in a bounded time with probability one, without any coordination between the sensors.

The paper is structured as follows. In the next section, we first give an overview of the scheme we propose to save energy consumption at the same time as to keep the latency bounded. We introduce formally the network model in Section 3. We begin by reminding a few useful facts about the classic static Poisson Boolean model, and we then extend it to account for nodes switching between on and off periods, in a dynamic model, which we name blinking Poisson Boolean model. We give some of their connectivity properties in the same section, and some of their coverage properties in Section 4. Section 5 is the main result of the paper, namely the proof that the latency of the linear grows linearly with the distance between the sink and the sensor that detected the event. We prove both a lower bound and an upper bound on this latency, using different percolation techniques: the upper bound is established using Liggett's ergodic theorem, while the lower bound is obtained by a coupling with the continuum growth model proposed by Deijfen [5]. The linearity of these bounds is also validated by simulation in Section 5.3, and the impact of the parameters of the network (node density, durations of on and off periods) is discussed.

\section{DECENTRALIZED ENERGY SAVING MECHANISM}

The general strategy we adopt to save energy and yet bound the latency of the network is as follows.

In the absence of any incoming event or message from a neighbour, nodes switch between their sleeping and active phases independently from each other. To save energy, we assume that the sleeping times are much longer than the active times.

Once a node has sensed an event, it stays active, and keeps sending repeatedly this information to its immediate neighbours, which can be reached within a single hop. The neighbours will only be able to hear the message when they turn their radio on. When all the neighbours have heard that message, the sensing node can turn off its radio, and resume its regular on/off schedule. In order to avoid synchronization, the phase of the on/off schedule is drawn according to the stationary distribution. The other nodes will then remain active until all their neighbours have received the message, and so on, until the sink eventually receives the message.

Remember that nodes are scattered over the domain that needs to be monitored, according to a Poisson process. We suppose that there is a large number of nodes, so that the sensing radius can be set to a small value without impacting the coverage of the network. However, since nodes switch between the two phases independently from each other, there may be quite few active nodes at any given time $t$, so that the set of simultaneously active nodes at any particular time $t$ is always disconnected. This set of simultaneously active nodes represents however only a snapshot of the network at this particular time. By waiting long enough, a sufficiently large number nodes will have passed by an active phase, so that the superposition of all the different snapshots of the network at all times within an interval becomes supercritical. On this cumulative graph, there is a path between any sensor and the sink with high probability. While it is relatively easy to show that indeed the network will pass by a supercritical phase if we wait long enough (as we will show in Proposition 2), it is much more difficult to find how long we have to wait before this path is almost surely present, that is, to compute the latency. Clearly, the larger the distance between the sensing node and the sink, the larger the latency. But how does it depend on this distance? The bounds on the latency, which we establish in Theorem 1, show that it is linear, under the conditions stated in the introduction. The simulations, run under more general conditions (including propagation delays on the links, and a random connectivity radius) confirm this finding.

\section{POISSON BOOLEAN MODELS}

\subsection{Static Poisson Boolean model}

In the static Poisson Boolean model, the locations of the "grains" are determined by points $\left\{X_{i}\right\}$ of a stationary Poisson point process in $\mathbb{R}^{d}$ of intensity $\lambda$ (see e.g. [24]). In this paper, we only consider $\mathbb{R}^{2}$ and disk shaped grains. Then the Poisson Boolean model $\mathscr{B}(\lambda, R)$ is just a union of randomly scattered disks (see Figure 1), i.e., the coverage process defines the occupied component

$$
\mathscr{B}(\lambda, R) \doteq \bigcup_{i} B\left(X_{i}, R_{i}\right),
$$

where $B\left(X_{i}, R_{i}\right)$ is the disk centered at $X_{i}$ having radius $R_{i}$, and where the $R_{i}$ are i.i.d., independent to point process $\left\{X_{i}\right\}$, and distributed as $R$
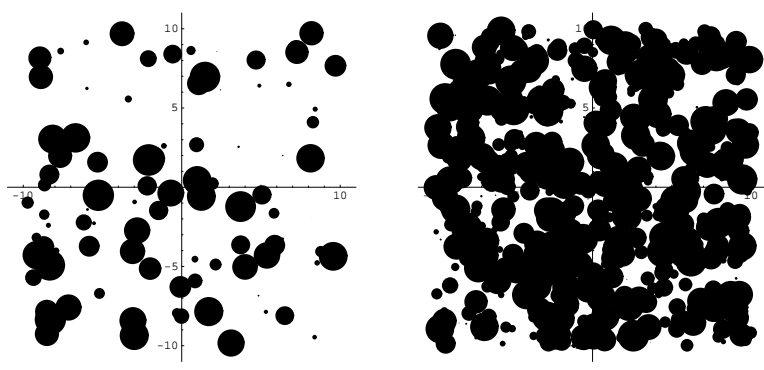

Figure 1: Poisson Boolean model in $\mathbb{R}^{2}$. Sub-critical intensity on the left and supercritical intensity on the right.

The coverage can be measured by the hitting probability, which is the probability that an arbitrary point belongs to the occupied region. In a stationary setting, the hitting probabilities are equal to the mean fraction of area occupied by $\mathscr{B}(\lambda, R)$. For the Poisson Boolean model, we have

$$
\begin{aligned}
\mathbb{P}(\mathbf{x} \in \mathscr{B}(\lambda, R)) & =\mathbb{P}(0 \in \mathscr{B}(\lambda, R))=1-e^{-\lambda \mathbb{E}\left(|B(0, R)|_{2}\right)} \\
& =1-\exp \left(-\lambda \pi \mathbb{E}\left(R^{2}\right)\right) .
\end{aligned}
$$

For more information see e.g. [24].

On the other hand, the occupied set can be divided into disjoint clusters which are formed by the overlapping disks. One way to measure the global connectivity is the size of the largest cluster. Let us denote $W(A), A \subset \mathbb{R}^{d}$, the union of all the occupied components which intersect $A$. Then, the critical intensity can be defined by checking if there is a positive probability that the origin belongs to an unbounded cluster, i.e.,

$$
\lambda_{c} \doteq \inf \left\{\lambda: \mathbb{P}_{\lambda}(\operatorname{diam}(W(\{0\}))=\infty)>0\right\} .
$$

Under quite weak assumptions ${ }^{1}$, in dimension $d \geq 2$, there exists $0<\lambda_{c}<\infty$ such that the largest connected component is unbounded a.s. whenever the intensity $\lambda>\lambda_{c}$. Moreover, the infinite cluster is unique a.s. On the other hand, if $\lambda<\lambda_{c}$, all the connected components are finite a.s. There is no analytical formula for $\lambda_{c}$, only bounds in some special cases. However, it is relatively

${ }^{1}$ E.g., $\mathbb{E}\left(R^{2 d-1}\right)<\infty$ 
easy to numerically estimate $\lambda_{c}$. For example, $\lambda_{c} r^{2} \approx 0.37$ for a fixed radius $R=r$, (see e.g. [25, 21]). For more details related to percolations in Poisson Boolean model see [18].

\subsection{Blinking Poisson Boolean model}

In order to take into account the alternation between the sleeping and active modes, we introduce a dynamic Poisson Boolean model, where the dynamics is due to the "blinking" of the nodes. As far as we know, only few dynamic percolation models have been researched mathematically. In the lattice, dynamic bond percolations have been studied by Häggström, Peres and Steif [14, 19]. Poisson Boolean models with moving points have been studied by van den Berg et al. [2].

The blinking Poisson Boolean model $\mathscr{B}(\lambda, R, Z)$ is a simple modification of the static Poisson Boolean model. As in the static Poisson Boolean model, the positions of the nodes are determined by a Poisson point process with intensity $\lambda$. At each node, we attach a disk whose radius is distributed as $R$ (deterministic or i.i.d. random radii). The dynamics follows from the assumption that the nodes alternate between on-state and off-state with periods determined by the stationary i.i.d. on/off processes $Z^{(i)}$. The distributions of these processes are equal to the distribution of process $Z$.

We assume that the lengths of the on/off periods are independent, with the off periods either constant or exponentially distributed with mean $t_{\text {off }}$, and the on periods distributed according to an arbitrary distribution with mean $t_{\text {on }}$. The only purely technical assumption we require for the on periods is that they have always nonzero length, which we can write as

$$
\lim _{\delta \rightarrow 0} \mathbb{P}\left[\min _{t \in[s, s+\delta)} Z_{t}=1 \mid Z_{s}=1\right]=1 .
$$

This is always the case in practice. On the other hand, assuming that $\delta<t_{\text {off }}$, the off periods satisfy

$$
\mathbb{P}\left[\max _{t \in[s, s+\delta)} Z_{t}=0 \mid Z_{s}=0\right]= \begin{cases}e^{-\delta / t_{\mathrm{off}}}, & \text { if exp-distributed, } \\ 1-\frac{\delta}{t_{\mathrm{off}}}, & \text { if constant. }\end{cases}
$$

The stationary distribution of $Z$ is given by

$$
\pi_{\text {off }} \doteq \mathbb{P}(Z=0)=\frac{t_{\text {off }}}{t_{\text {off }}+t_{\text {on }}} \quad \text { and } \quad \pi_{\text {on }} \doteq \mathbb{P}(Z=1)=\frac{t_{\text {on }}}{t_{\text {off }}+t_{\text {on }}} \text {. }
$$

Assume that the node density is supercritical, that is, $\lambda>\lambda_{c}$. From the point of view of sensor networks, it is interesting to know whether the network remains connected all the time, despite the alternation between sleeping and active states. If there is no unbounded connected component of active nodes at any snapshot, then another question is how long one has to wait in order to get a completely connected network in the cumulative coverage process. The two situations are visualized in Figure 2 and answers are given by the following propositions.

Proposition 1. Assume $\lambda>\lambda_{c}$. If the $Z^{(i)}$ are stationary on/off processes with exponential or constant off times and on periods satisfying (1), then

$$
\begin{gathered}
\mathbb{P}(\text { an infinite cluster exists for all } t \geq 0)=1 \text {, if } \lambda_{c}<\lambda \pi_{\mathrm{on}}, \\
\mathbb{P}(\text { there is no infinite cluster for all } t \geq 0)=1 \text {, if } \lambda_{c}>\pi_{\mathrm{on}} \lambda .
\end{gathered}
$$

PRoOF. A slightly modified proof from [14]. Let us assume first $\lambda \pi_{\mathrm{on}}>\lambda_{c}$. Let $\varepsilon>0$ such that $(1-\varepsilon) \pi_{\mathrm{on}}>\lambda_{c} / \lambda$ and take
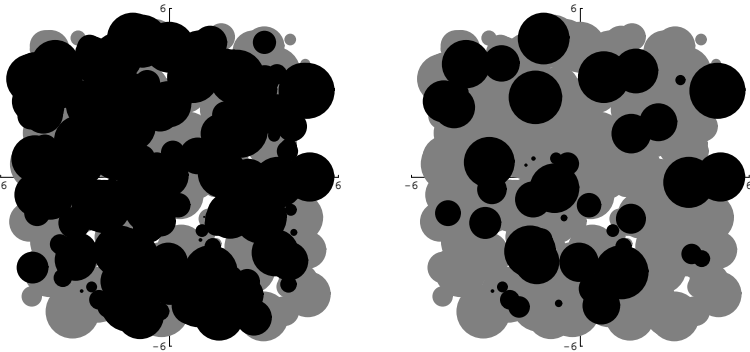

Figure 2: Snapshots of the Blinking Poisson Boolean model at some particular time $t$. The black disks are the coverage area of the active nodes, the gray ones of the sleeping nodes. On the left $\lambda>\pi_{\mathrm{on}} \lambda>\lambda_{c}$, i.e., there is always an unbounded active cluster. On the right, $\pi_{\mathrm{on}} \lambda<\lambda_{c}<\lambda$, i.e., all the active clusters are finite a.s.

$\delta>0$ such that $\mathbb{P}\left[\min _{t \in[0,0+\delta]} Z_{t}=1 \mid Z_{0}=1\right]>1-\varepsilon$. Then $p_{\delta} \doteq \mathbb{P}\left(\min _{t \in[0, \delta]} Z_{t}^{(i)}=1\right)>(1-\varepsilon) \pi_{\mathrm{on}}>\lambda_{c} / \lambda$ for an arbitrary $i$. Since events $\left\{\inf _{t \in[0, \delta]} Z_{t}^{(i)}=1\right\}, i=1,2, \ldots$, are mutually independent, we can consider a thinning of a Poisson process where we take only the nodes which are active the whole interval $[0, \delta]$. These nodes are distributed according to a Poisson process with intensity $p_{\delta} \lambda>\lambda_{c}$. Thus $\mathbb{P}($ an infinite cluster exists for all $t \in[0, \delta])=1$. The argument can be repeated for the intervals $[k \delta,(k+1) \delta]$ with integer $k$. Denote $E_{k}$ the event that an infinite cluster exists for all $t \in[k,(k+1) \delta]$, and $E_{k}^{c}$ its complement. Then

$$
\mathbb{P}\left(\bigcap_{k} E_{k}\right)=1-\mathbb{P}\left(\bigcup_{k} E_{k}^{c}\right) \geq 1-\sum_{k} \mathbb{P}\left(E_{k}^{c}\right)=1 .
$$

If $\lambda \pi_{\text {on }}<\lambda_{c}$, then we consider the nodes which are not sleeping during the whole interval $[0, \delta]$. By Equation (2), for small enough $\delta, \mathbb{P}\left(\max _{t \in[0, \delta]} Z_{t}=1\right)<\pi_{\text {on }}+\pi_{\text {off }} \delta / t_{\text {off }}<\lambda_{c} / \lambda$. Thus the intensity of those points is less than the critical intensity and there is a.s. no unbounded cluster. Again, the countable additivity completes the proof.

Even if $\pi_{\mathrm{on}} \lambda<\lambda_{c}$ we can still a have some form of connectivity if we take into account the cumulative coverage process. Let us define the cumulative Blinking Boolean model as the the area which has been covered by some active disk within $[0, t]$.

Proposition 2. Assume $\lambda>\lambda_{c}$. If the $Z^{(i)}$ are i.i.d. stationary on/off processes with exponential off-times, then the cumulative connectivity graph on $[0, t]$ has almost surely an infinite connected component whenever $t>t_{\mathrm{off}} \log \frac{\lambda-\lambda_{c}}{\lambda-\lambda \pi_{\mathrm{on}}}$. If the off periods last constant time then the condition is $t>t_{\mathrm{off}} \frac{\lambda_{c}-\lambda \pi_{\mathrm{on}}}{\lambda-\lambda \pi_{\mathrm{on}}}$.

Proof. The probability that process $Z$ visits at least once the on-state within $[0, t]$ is

$$
\begin{aligned}
& \mathbb{P}\left(\max _{s \in[0, t]} Z_{s}=1\right) \\
& \quad=\mathbb{P}\left(Z_{0}=1\right)+\mathbb{P}\left[\max _{s \in[0, t]} Z_{s}=1 \mid Z_{0}=0\right] \mathbb{P}\left(Z_{0}=0\right) \\
& \quad=1-\pi_{\text {off }} \mathbb{P}\left[\max _{s \in[0, t]} Z_{s}=0 \mid Z_{0}=0\right] .
\end{aligned}
$$


The points of the Poisson process, where the corresponding on/off processes visit the on-state during $[0, t]$, form a thinned Poisson point process with intensity $1-\pi_{\mathrm{off}} \mathbb{P}\left[\max _{s \in[0, t]} Z_{s}=0 \mid Z_{0}=0\right]$. The corresponding Poisson Boolean model has an unbounded cluster a.s. if $\lambda\left(1-\pi_{\text {off }} \mathbb{P}\left[\max _{s \in[0, t]} Z_{s}=0 \mid Z_{0}=0\right]\right)>\lambda_{c}$. In order to complete the proof, apply Equation (2).

The next section shows how these results are useful for determining the coverage and connectivity of the network.

\section{SENSING COVERAGE AND TRANSMIS- SION CONNECTIVITY}
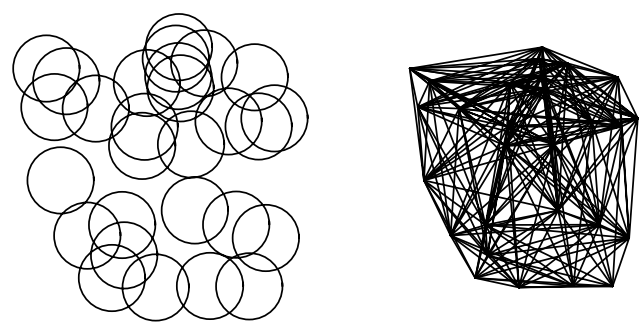

Figure 3: A sensing coverage on the right and the corresponding transmission network on the right when transmission radius is 5 times bigger than the sensing radius.

We assume that the radio equipments are alternating between sleep and active states with mean sojourn times $t_{\text {off }}$ and $t_{\text {on }}$ and stationary distributions $\pi_{\mathrm{off}}$ and $\pi_{\mathrm{on}}$. The sensing apparatus are either active all the time or they also interchange according to some on/off processes, with parameters $t_{\mathrm{off}}^{s}$ and $t_{\mathrm{on}}^{s}$ and stationary distributions $\pi_{\text {off }}^{s}$ and $\pi_{\text {on }}^{s}$.

For the sensing model, we let the radii be random, but for the transmission model the radii are assumed to be constant and denoted by $r_{\text {rad }}=2 r$. Note that if two disks of radius $r$ overlap, then the centers are at most $2 r$ apart. Thus the radio connectivity graph is determined by Boolean model with disks of radius $r$.

\subsection{Sensing area}

For some networks, the covered fraction of the area is the key property, for others the probability of unwatched routes through the network. Thus, depending on the application, either the mean area coverage or the existence of a percolation cluster are the characteristics which give the conditions for the minimal density of the sensors.

Assume that each sensor can monitor a disk whose radius is distributed as $R$. As explained in the previous section, the mean fraction of area covered is given by $1-\exp \left(-\lambda \pi_{\mathrm{on}}^{s} \mathbb{E}\left(R^{2}\right)\right)$. To ensure a covered fraction at least equal to $p_{A}$, the average number of active sensors per unit area $\lambda \pi_{\mathrm{on}}^{s}$ has to satisfy $\lambda \pi_{\mathrm{on}}^{s} \geq \frac{-1}{\pi \mathbb{E}\left(R^{2}\right)} \log \left(1-p_{A}\right)$.

Regarding unwatched routes, percolation theory gives the critical intensities guaranteeing that there is an unbroken net guarding any passage through the network. To ensure a connected covered area at all instants, it is enough to have $\lambda \pi_{\mathrm{on}}^{s}>\lambda_{c}$, according to Proposition 1.

If alarms are triggered by, for example, slowly moving events, then it is enough to obtain connectivity over a longer time period. In this case, Proposition 2 gives the sufficient conditions with respect to the intensity and the mean lengths of the on/off periods. More details on the coverage properties of sensor networks can be found in [17].

\subsection{Radio connectivity}

Connectivity can be seen as the probability that an arbitrary node is connected to most of the others.

If we assume that the radio range of the devices is significantly larger than their sensing range, the conditions on the node density given in the above section lead to a highly super-critical radio connectivity graph (see Figure 3). In fact, almost all the nodes are connected in this case, and there exist highly redundant routes between nodes. This redundancy is the motivation for letting the nodes turn off their radio device sporadically.

In our mechanism, at each time instant, the number of nodes with active radio device is $\pi_{\mathrm{on}} \lambda$. This defines a new static Poisson Boolean model $\mathscr{B}\left(\pi_{\mathrm{on}} \lambda, r\right)$. Depending on the value of $\pi_{\mathrm{on}}$, the new model can be either super-critical, either sub-critical, as shown in Proposition 1. In order to spare as much battery as possible, in this paper, we choose $\pi_{\text {on }}$ so low that the resulting process is sub-critical. Even in this case, messages can be carried from almost any node of the network to almost any other node. More precisely, messages can be exchanged between any two nodes that belong to the infinite cluster of $\mathscr{B}(\lambda, r)$. The transmission latency with respect to the distance between source and destination nodes is studied in detail in Section 5. The case where $\pi_{\text {on }}$ is high enough to keep the network super-critical at all times will be addressed in future research.

\section{LATENCY}

Assume that the node sensing the incoming event is placed at the origin. This node starts sending an alarm message at time 0. All the active nodes, within transmission radius $2 r$ or less, receive the message and they also begin to broadcast over their own transmission areas. Assuming no propagation delays, at $t=0$ the message has spread to the cluster containing the origin (see Figure 4a). A sleeping node inside the set that was already covered by the broadcast message and that changes its state is called a bridge After the bridge has started its broadcast, all the new active nodes who receive the message, either directly from the bridge or via a multihop path, are added to the original cluster (see Figure $4 \mathrm{~b}$ ). If the origin belongs to the infinite cluster of $\mathscr{B}(\lambda, r)$ the process continues forever, otherwise it stops after finitely many steps. a)

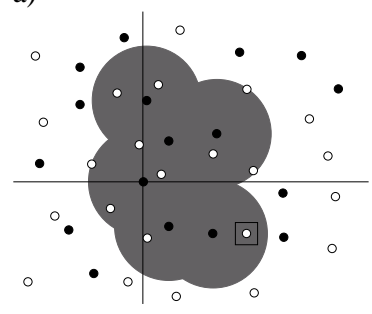

b)

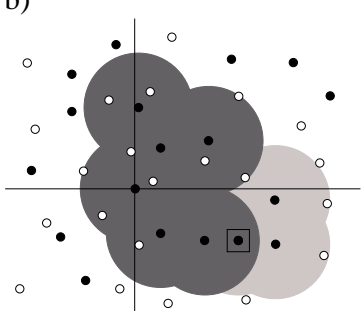

Figure 4: Message spreading is shown in gray area. In this picture the disk radii are $r_{\text {rad }}=2 r$. White nodes are sleeping, black nodes active and a bridge is indicated by the box.

\subsection{Linear spreading of alarm messages}

In this section we state our main result. Assume that alarm messages are transmitted over a blinking Poisson Boolean model $\mathscr{B}(\lambda, r, Z)$. Remember that means assuming a constant transmission radius $2 r$ for each sensor. First, we show that if an alarm occurs in a random place inside the infinite connected component of $\mathscr{B}(\lambda, r)$, the latency is asymptotically linear with respect to the dis- 
tance to the sink. Secondly, the maximum distance from where the alarm message can be heard is also behaving asymptotically linearly.

These results allow us to dimension the transmission part of a sensor network. First of all, they give us tools to tune up the alternation between sleep and active phase so that the speed of alarm detection meets the predefined requirements. In addition, if one wants to analyze the interference due to separate alarms appearing about the same time, maximum message distance can be used to approximate the area where the messages collide.

Assume that two nodes, located at $X$ and $Y$, belong to the infinite cluster of $\mathscr{B}(\lambda, r)$. Denote by $T(X, Y)$ the time it takes to transmit an alarm message from $X$ to $Y$. The first part of Theorem 1 shows that $T(X, Y)$ is asymptotically linear in distance $|X-Y|$. In the second scenario, we fix only the source $X$, and denote by $G_{t}^{X}$ the nodes which have received the message at time $t . G_{t}^{X}$ is called the message cluster. Then $\max \left|G_{t}^{X}\right|=\max \left\{|y-X|: y \in G_{t}\right\}$ measures the maximal transmission distance. This value is shown to be also asymptotically linear.

THEOREM 1. If two nodes, located at $X$ and $Y$, belong to the infinite cluster of the Poisson Boolean model $\mathscr{B}(\lambda, r)$, resulting from a blinking Poisson Boolean model $\mathscr{B}(\lambda, r, Z)$ with $\lambda>\lambda_{c}>\pi_{\mathrm{on}} \lambda$ and $Z$ stationary on/off process with exponential or constant offtimes, then there is a finite strictly positive constant $\eta$ such that

$$
(1-\varepsilon) \eta \leq \frac{T(X, Y)}{|X-Y|} \leq(1+\varepsilon) \eta
$$

for any $\varepsilon>0$ whenever $|X-Y|$ is large enough. Moreover, there are finite strictly positive constants $\mu$ and $\bar{\mu}$ such that

$$
\underline{\mu} \leq \frac{\max \left|G_{t}^{X}\right|}{t} \leq \bar{\mu}
$$

whenever $t$ is large enough.

We will prove this theorem in the two following subsections. The linearity of the transmission time between a given pair of points is based on Liggett's subadditive ergodic theorem. The linear growth of the message cluster is proved by coupling it with a continuum growth model.

It is important to notice that the constant $\eta$ only depends on the parameters of the network - namely $\lambda, r, t_{\mathrm{on}}$ and $t_{\mathrm{off}}$ - but not on the random disposition of the nodes. This value can thus be estimated by simulation, given a set of parameters, and used to predict the performance of the network before its deployment.

\subsubsection{Proof of Equation (3)}

We consider first passage percolation in the random graph determined by $\mathscr{B}(\lambda, r)$. Assume that the random variables $T_{i}$ satisfy

$$
\begin{aligned}
\mathbb{P}\left(T_{i}=0\right) & =\pi_{\text {on }}, \\
\mathbb{P}\left(T_{i}>t\right) & =\pi_{\text {off }} \mathbb{P}\left(Z_{s}=0 \forall s \in[0, t] \mid Z_{0}=0\right),
\end{aligned}
$$

i.e., $T_{i}$ is the time until a node inside the message cluster turns active. If needed, the propagation delays could also be included in the random variables $T_{i}$. To each oriented edge $\left(X_{i}, X_{j}\right)$ of the random graph, we attach a time coordinate (or "delay") $T_{j}$. It is easy to couple the models in such a way that the message transmission time in a blinking Poisson Boolean model and the first passage time in the weighted graph are equal, i.e.,

$$
T(X, Y)=\inf _{w(X, Y)}\left\{\sum_{X_{i} \in w(X, Y)} T_{i}\right\}
$$

where $w(X, Y)$ is an arbitrary path joining $X$ and $Y$. However, notice that the other paths, except the fastest connection, may arise later in the static delay model. In the blinking model, when a node turns active inside the message cluster more than one new link can join the connectivity graph.

Without losing generality, we consider the first passage percolation in the direction of the $x$-axis. For any $(x, 0) \in \mathbb{R}^{2}$, we denote the index of the nearest node in the infinite cluster $C_{\infty}$ by $i(x)=$ $\operatorname{argmin}_{i}\left\{\left|(x, 0)-X_{i}\right|: X_{i} \in C_{\infty}\right\}$. Let $\tilde{X}(x)=X_{i(x)}$ and $\tilde{T}(x, y)=$ $T(\tilde{X}(x), \tilde{X}(y))$ (see Figure 5). Next define the collection of indexed variables by $T_{m, n}=\tilde{T}(m x, n x)$, for some constant $x>0$

Using Liggett's subadditive ergodic theorem (Theorem 2), we can prove the following proposition.

PROPOSITION 3.

$$
\lim _{n \rightarrow \infty} \frac{\tilde{T}(0, n x)}{n}=\lim _{n \rightarrow \infty} \frac{T_{0, n}}{n}=\eta(x) \quad \text { a.s. }
$$

where $\eta(x)=\inf _{n \geq 1} \mathbb{E}(\tilde{T}(0, n x)) / n$.

THEOREM 2. [16, Liggett's subadditive ergodic theorem] Let $\left\{T_{m, n}\right\}$ be a collection of random variables indexed by integers satisfying $0 \leq m<n$. Suppose $\left\{T_{m, n}\right\}$ has the following properties:

(i) $T_{0, n} \leq T_{0, m}+T_{m, n}$.

(ii) For each $n, \mathbb{E}\left(\left|T_{0, n}\right|\right)<\infty$ and $\mathbb{E}\left(T_{0, n}\right) \geq$ cn for some constant $c>-\infty$.

(iii) The distribution of $\left\{T_{m, m+k}: k \geq 1\right\}$ does not depend on $m$.

(iv) For each $k \geq 1,\left\{T_{n k,(n+1) k}: n \geq 0\right\}$ is a stationary sequence.

Then:

(a) $\eta \doteq \lim _{n \rightarrow \infty} \mathbb{E}\left(T_{0, n}\right) / n=\inf _{n \geq 1} E\left(T_{0, n}\right) / n$.

(b) $T \doteq \lim _{n \rightarrow \infty} T_{0, n} / n$ exists a.s.

(c) $\mathbb{E}(T)=\eta$

Furthermore, if $k \geq 1,\left\{T_{n k,(n+1) k}: n \geq 0\right\}$ are ergodic, then

(d) $T=\eta$ a.s.

As $T_{0, m}$ is the first passage time from $\tilde{X}(0)$ to $\tilde{X}(m x)$, and $T_{m, n}$ the passage time from $\tilde{X}(m x)$ to $\tilde{X}(n x)$, it is clear that $T_{0, n}$ is at most $T_{0, m}+T_{m, n}$. Condition ( $\left.i\right)$ is thus verified.

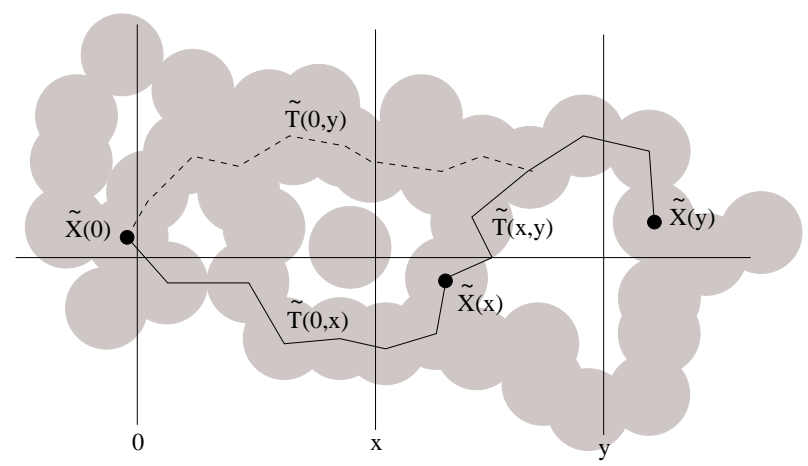

Figure 5: First passage percolation paths. The routes correspond to the fastest paths between points $\tilde{X}(0), \tilde{X}(x)$ and $\tilde{X}(y)$.

As a first passage time cannot be negative, we have $\mathbb{E}\left(T_{m, n}\right) \geq 0$, $\forall m, n$. To compute an upper bound of $\mathbb{E}\left(T_{m, n}\right)$, we consider the 


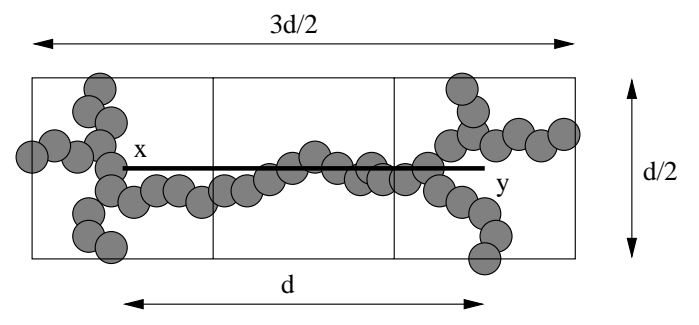

Figure 6: A good rectangle associated with a horizontal edge. It contains a left-right crossing and a top-bottom crossings in both sub-squares at its ends

shortest path (in distance) from $\tilde{X}(m x)$ to $\tilde{X}(n x)$ in $\mathscr{B}(\lambda, r)$. A suboptimal strategy is to follow this path, and wait at each step that the next hop becomes active. In the worst case, the message has to wait in average $t_{\text {off }}$ seconds at each step. We have thus

$$
\mathbb{E}\left(T_{m, n}\right) \leq t_{\text {off }} \mathbb{E}\left(L_{\tilde{X}(m x), \tilde{X}(n x)}\right),
$$

where $L_{\tilde{X}(m x), \tilde{X}(n x)}$ denotes the length in hops of the shortest path between $\tilde{X}(m x)$ and $\tilde{X}(n x)$. Proposition 4 will ensure that the latter expected value is always finite, and thus that Condition (ii) is verified. To prove this proposition, we need the following two preliminary lemmas.

LEMMA 1. (See e.g. [12] p. 295) In an independent bond percolation model of open edge density $\tilde{p}>1 / 2$, we denote by $A_{n}$ the event that there exists an open path in the rectangle $R_{n}=[0,4 n] \times$ $[0, n]$ that joins its left and right borders (left-right crossing). There exist constants $\alpha<\infty$ and $\gamma>0$ such that $\mathbb{P}_{\tilde{p}}\left(A_{n}\right) \geq 1-\alpha n e^{-\gamma n}$.

LEMMA 2. In $\mathscr{B}(\lambda, r)$, we denote by $C_{n}$ the event that the origin is surrounded by an occupied circuit (i.e. a circuit entirely included in the occupied region) that is contained in the frame $F_{n}=$ $([-n, n] \times[-n, n]) \backslash([-n / 2, n / 2] \times[-n / 2, n / 2])$. There exist constants $\alpha<\infty$ and $\gamma>0$ such that $\mathbb{P}\left(C_{n}\right) \geq 1-\alpha n e^{-\gamma n}$.

Proof. We use a renormalization argument to map the Boolean model to a discrete model. We start by constructing a square lattice over the plane, with edge length $d$. For each edge $(x, y)$ of the lattice, with $y=x+(d, 0)$ or $y=x+(0, d)$, we consider the rectangle $\left[x_{1}-d / 4, y_{1}+d / 4\right] \times\left[x_{2}-d / 4, y_{2}+d / 4\right]$, as depicted in Figure 6 . We call a horizontal rectangle good if there exist in $\mathscr{B}(\lambda, r)$ an open cluster that crosses it from left to right, an open cluster that crosses $\left[x_{1}-d / 4, x_{1}+d / 4\right] \times\left[x_{2}-d / 4, x_{2}+d / 4\right]$ from bottom to top, and an open cluster that crosses $\left[y_{1}-d / 4, y_{1}+d / 4\right] \times\left[y_{2}-d / 4, y_{2}+\right.$ $d / 4]$ from bottom to top. We define good vertical rectangles in the same way, except that we exchange left-right with top-bottom. As $\mathscr{B}(\lambda, r)$ is supercritical, for any probability $p<1$, one can choose $d$ large enough so that a rectangle is good with probability at least $p$ (see [18, Corollary 4.1]).

We then declare an edge open if it is surrounded by a good rectangle, and closed otherwise. We obtain thus a dependent bond percolation model. However, if two edges have no common vertex, their states are independent. Our model is thus a 1-dependent percolation model, which is known to percolate if $p$ is large enough. More precisely, one can find a product measure $\mu_{\tilde{p}}$ on this model, where each edge is open with probability $\tilde{p}>1 / 2$, that is stochastically dominated by our 1-dependent measure $\mu_{p}$ [12].

We assume without loss of generality that the vertices of the lattice have the form $(i, j)$, for $i, j \in \mathbb{Z}$. In the bond percolation model a)

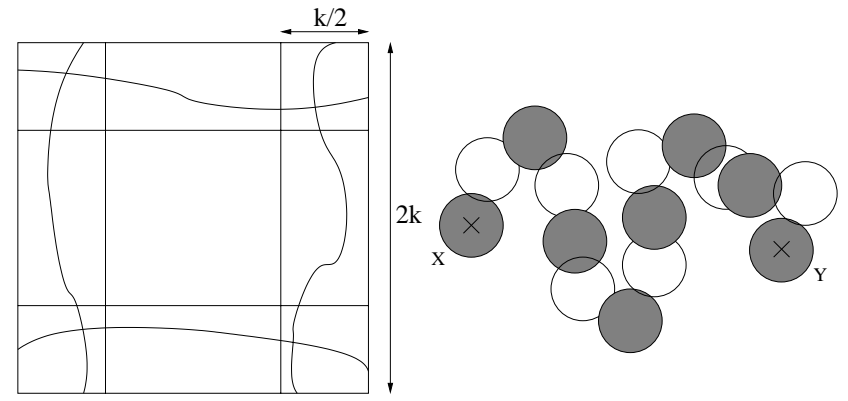

Figure 7: a) A frame that surrounds the origin. The four crossings form a circuit. b) The shortest path from $X$ to $Y$. It is made of a chain of balls, that cannot overlap any other ball than their predecessor and successor.

with product measure, we look for left-right crossings in the rectangles $[-k, k] \times[-k,-k / 2]$ and $[-k, k] \times[k / 2, k]$ (their actual size is thus $2 k d \times k d / 2$ ) for some $k \in \mathbb{N}$. The probability that such a crossing exists is given by Lemma 1 . The same is true for topbottom crossings in $[-k,-k / 2] \times[-k, k]$ and $[k / 2, k] \times[-k, k]$. The probability that there is a crossing in each of these four rectangles simultaneously is bounded by

$$
\mathbb{P}_{\mu_{\tilde{p}}}(4 \text { crossings }) \geq 1-4 \alpha^{\prime} n e^{-\gamma^{\prime} k} .
$$

As these crossings overlap at each corner, the origin is surrounded by an open circuit that is contained in

$$
([-k, k] \times[-k, k]) \backslash([-k / 2, k / 2] \times[-k / 2, k / 2])
$$

(see Figure 7a). Since the product measure $\mu_{\tilde{p}}$ is dominated by the 1-dependent measure, the crossings appear with higher or equal probability than in the independent case. Therefore,

$$
\mathbb{P}_{\mu_{p}}(4 \text { crossings }) \geq \mathbb{P}_{\mu_{\tilde{p}}}(4 \text { crossings }) .
$$

Moreover, our construction is such that the existence of an open circuit in the discrete model implies that $\mathscr{B}(\lambda, r) \cap([-k d, k d] \times$ $[-k d, k d]) \backslash([-k d / 2, k d / 2] \times[-k d / 2, k d / 2])$ contains a component that surrounds the origin, which is the event we want. The final result is obtained by letting $\alpha=4 \alpha^{\prime}$ and $\gamma=\gamma^{\prime} / d$ in (5).

Proposition 4. If $X$ and $Y$ are two points of a Boolean model, located at finite distance, that belong to the same cluster, and let $L_{X Y}$ be the number of hops in the shortest path between them. Then $\mathbb{E}\left(L_{X Y}\right)$ is finite.

Proof. We assume without loss of generality that $X=(x, y)$ and $Y=(-x,-y)$, and consider a frame $F_{n}$, as defined in Lemma 2 , with $n>2 \sqrt{x^{2}+y^{2}}$. If this frame contains an occupied circuit, then the shortest path from $X$ to $Y$ is included in the square $[-n, n] \times$ $[-n, n]$.

On the other hand, the shortest path is made of a chain of balls of radius $r$, and it is impossible that a ball overlaps more than two other balls of the path. Otherwise, one could remove one ball and shorten the path. Therefore, a $\ell$ hops path must contain at least $\ell / 2$ disjoint balls (see Figure 7b). The surface occupied by the path is thus at least $\pi r^{2} \ell / 2$. As the path is contained in a square of surface $4 n^{2}$, the length of the path cannot exceed $\ell \leq \frac{8 n^{2}}{\pi r^{2}}$. Therefore, $\mathbb{P}\left(L_{X Y} \leq \frac{8 n^{2}}{\pi r^{2}}\right) \geq \mathbb{P}\left(C_{n}\right)$. Combining this with Lemma 2 gives 
$\mathbb{P}\left(L_{X Y}>\frac{8 n^{2}}{\pi r^{2}}\right) \leq 1-\mathbb{P}\left(C_{n}\right) \leq \alpha n e^{-\gamma n}$. Thus, we can find $m$ large enough so that for $k \geq m, \mathbb{P}\left(L_{X Y}>k\right) \leq \alpha\lfloor r \sqrt{k \pi / 8}\rfloor e^{-\gamma\lfloor r \sqrt{k \pi / 8}\rfloor}$. We can finally upper bound the expected value of the length of the shortest path:

$$
\begin{aligned}
\mathbb{E}\left(L_{X Y}\right) & =\sum_{k=0}^{\infty} \mathbb{P}\left(L_{X Y}>k\right) \\
& =\sum_{k=0}^{m-1} \mathbb{P}\left(L_{X Y}>k\right)+\sum_{k=m}^{\infty} \mathbb{P}\left(L_{X Y}>k\right) \\
& \leq m+\sum_{k=m}^{\infty} \alpha\lfloor r \sqrt{k \pi / 8}\rfloor e^{-\gamma\lfloor r \sqrt{k \pi / 8}\rfloor}<\infty .
\end{aligned}
$$

Conditions (iii) and (iv) are clearly verified, as $T_{m, n}$ is defined in a stationary way. The following lemma is to prove that the sequence $\left\{T_{n, n+1}\right\}$ is ergodic. In fact, we show that it is mixing (i.e., roughly speaking, asymptotically independent), which is a stronger property.

\section{Lemma 3 . The sequence $\left\{T_{n, n+1}\right\}, n \geq 0$ is mixing.}

PROOF. We compute $T_{0,1}$ by the following construction: we consider the square $B_{k}$ of edge length $k x$ centered at the origin. We denote by $C_{k}$ the largest occupied connected component of $B_{k} \cap \mathscr{B}(\lambda, r)$, and by $\tilde{X}^{(k)}(y)=X_{\operatorname{argmin}_{i}}\left\{\left|(y, 0)-X_{i}\right|: X_{i} \in C_{k}\right\}$ the closest point to $(y, 0)$ of $C_{k}$. We then define $T_{0,1}^{(k)}$ as the transmission time from $\tilde{X}^{(k)}(0)$ to $\tilde{X}^{(k)}(x)$. We observe that when $k$ goes to infinity, as $\mathscr{B}(\lambda, r)$ is supercritical, the largest occupied component in $B_{k}$ is $C_{\infty} \cap B_{k}$. Moreover, as a consequence of Proposition 4, the shortest path between $\tilde{X}^{(k)}(0)$ and $\tilde{X}^{(k)}(x)$ is finite. Thus we have that $\lim _{k \rightarrow \infty} T_{0,1}^{(k)}=T_{0,1}$ almost surely and therefore,

$$
\lim _{k \rightarrow \infty} \mathbb{P}\left(T_{0,1}^{(k)}<t\right)=\mathbb{P}\left(T_{0,1}<t\right), \forall t \in \mathbb{R} .
$$

We consider now the translation $S_{(x, 0)}$ over the vector $(x, 0)$ in $\mathbb{R}^{2}$. Clearly we have $S_{(x, 0)}^{n}\left(T_{0,1}\right)=T_{n, n+1}$. Similarly, we define $T_{n, n+1}^{(k)}:=S_{(x, 0)}^{n}\left(T_{0,1}^{(k)}\right)$. The same property is true for the translated variables

$$
\lim _{k \rightarrow \infty} \mathbb{P}\left(T_{n, n+1}^{(k)}<t^{\prime}\right)=\mathbb{P}\left(T_{n, n+1}<t^{\prime}\right),
$$

and for combinations of two events

$\lim _{k \rightarrow \infty} \mathbb{P}\left(\left(T_{0,1}^{(k)}<t\right) \cap\left(T_{n, n+1}^{(k)}<t^{\prime}\right)\right)=\mathbb{P}\left(\left(T_{0,1}<t\right) \cap\left(T_{n, n+1}<t^{\prime}\right)\right)$.

Finally, we can show that the sequence $\left\{T_{n, n+1}\right\}$ is mixing by setting $n=2 k$ :

$$
\begin{aligned}
& \lim _{k \rightarrow \infty} \mathbb{P}\left(\left(T_{0,1}<t\right) \cap\left(T_{2 k, 2 k+1}<t^{\prime}\right)\right) \\
& =\lim _{k \rightarrow \infty} \mathbb{P}\left(\left(T_{0,1}^{(k)}<t\right) \cap\left(T_{2 k, 2 k+1}^{(k)}<t^{\prime}\right)\right) \\
& =\lim _{k \rightarrow \infty} \mathbb{P}\left(T_{0,1}^{(k)}<t\right) \mathbb{P}\left(T_{2 k, 2 k+1}^{(k)}<t^{\prime}\right) \\
& =\mathbb{P}\left(T_{0,1}<t\right) \mathbb{P}\left(T_{2 k, 2 k+1}<t^{\prime}\right), \forall t, t^{\prime} \in \mathbb{R} .
\end{aligned}
$$

The second equality follows from the fact that $T_{0,1}^{(k)}$ and $T_{2 k, 2 k+1}^{(k)}$ are independent, as they depend on the realization of the blinking Boolean model on two disjoint squares.
Now we have seen that $T_{m, n}$ satisfies all the conditions of Theorem 2 and thus proved Proposition 3. Proposition 5 presented in the next section ensures that $\eta(x)>0$.

Finally, we should show that $\Delta^{n x}=\tilde{X}(n x)-(n x, 0)$ does not play any role asymptotically and that the discrete limit can be replaced by a continuous one. Although both claims are quite evident, we give a short sketch of their proof.

LEMMA 4. $\lim _{y \rightarrow \infty} \frac{T(\tilde{X}(0), \tilde{X}(y))}{|\tilde{X}(0)-\tilde{X}(y)|}=\eta$ a.s.

PROOF. (Sketch) For independent bond percolation with $p>$ $p_{c}\left(Z^{d}\right), d \geq 2$, there exists $\alpha>0$ such that $\mathbb{P}\left(B_{k} \cap C_{\infty}=\emptyset\right) \leq e^{-\alpha k}$ (see e.g. [13]). Using Borel-Cantelli and the same mapping from Poisson Boolean model to discrete percolation as in Lemma 2, shows that $\Delta^{n x} / n x \rightarrow 0$ a.s.

Consider rational $t_{n}=n q=n k / m, k, m \in \mathbb{Z}^{+}$and denote $\bar{T}_{t}=$ $\frac{\tilde{T}(0, t x)}{t}$. Then $\bar{T}_{n k} \rightarrow \eta(x)$, since $\left\{\bar{T}_{n k}\right\}$ is a subsequence of $\left\{\bar{T}_{n}\right\}$ which converges to $\eta(x)$ by Proposition 3. Also by Proposition 3 , $\frac{\tilde{T}(0, n q x)}{n} \rightarrow \eta(q x)$ a.s. Thus $\bar{T}_{n q} \rightarrow \eta(q x) / q$ a.s. Since $\left\{\bar{T}_{n k}\right\} \subset$ $\left\{\bar{T}_{n q}^{n}\right\}$, they have the same limit, i.e., $\eta(q x) / q=\eta(x)$. Thus

$$
\lim _{t_{n} \rightarrow \infty} \frac{\tilde{T}\left(0, t_{n} x\right)}{t_{n} x}=\eta(1) \doteq \eta \quad \text { a.s. }
$$

\subsubsection{Proof of Equation (4)}

The lower bound in Inequality (4) follows directly from (3). The next proposition gives the upper bound. Assuming a source at the origin, let $G_{t}$ denote the nodes which have received the message at time $t$ and $S_{t}$ the area covered by these nodes if a disk of radius $2 r$ is attached into each of them. Naturally, $G_{t} \subset S_{t}$.

Proposition 5. Consider the blinking Poisson Boolean model $\mathscr{B}(\lambda, r, Z)$ with $\lambda>\lambda_{c}$. If $\pi_{\mathrm{on}} \lambda<\lambda_{c}$, then there is $\mu>0$ such that almost surely $\frac{\max \left|S_{t}\right|}{t} \leq \mu$ for all sufficiently large $t$.

The claim is proved by showing that $S_{t}$ can be bounded above by a continuum growth model (see Appendix A) which is driven by a Poisson point process with intensity $\lambda \pi_{\mathrm{off}} / t_{\mathrm{off}}$ and an exponentially bounded disk size distribution.

Set $T_{0}=0$. At time epochs $T_{i}>0, i=1,2, \ldots$ one of the offnodes inside $S_{T_{i-1}}$, denoted by $X_{i}$, changes its state and $S_{t}$ possibly grows. $C\left(X_{i}\right) \doteq S_{T_{i}} \backslash S_{T_{i-1}}$ determines the new area which receives the message at time $T_{i}$ (shown in light gray in Figure 8). We will first show that $C\left(X_{i}\right)$ can be bounded by disks $B\left(X_{i}, D_{i}\right)$ with i.i.d. $D_{i}$. This follows from the cluster size distribution in a subcritical regime of a Poisson Boolean model.

Let $A \leadsto B$ denote the event that sets $A$ and $B$ intersect the same cluster.

Lemma 5. [18, Theorem 2.4] Consider Poisson Boolean model $\mathscr{B}(\lambda, R)$ where $R$ satisfies $0 \leq R \leq r$ for some $r<\infty$. Assuming $\mathbb{E}(\operatorname{diam}(W(\{0\})))<\infty$ then there exist positive constants $C_{1}$ and $C_{2}$, depending on $\lambda$ and the dimension $d$, such that

$$
\mathbb{P}\left(S \sim B(0, m)^{c}\right)<C_{1} \exp \left(-C_{2} m\right)
$$

for an arbitrary bounded set $S$.

LEMMA 6. If $\pi_{\mathrm{on}} \lambda<\lambda_{c}$, then $C\left(X_{i}\right) \subseteq B\left(X_{i}, D_{i}\right)$ a.s., where $D_{i}$ are i.i.d. with

$$
\mathbb{P}\left(D_{i}>a\right)<C_{1} \exp \left(-C_{2} a\right)
$$

for some positive constants $C_{1}$ and $C_{2}$. 


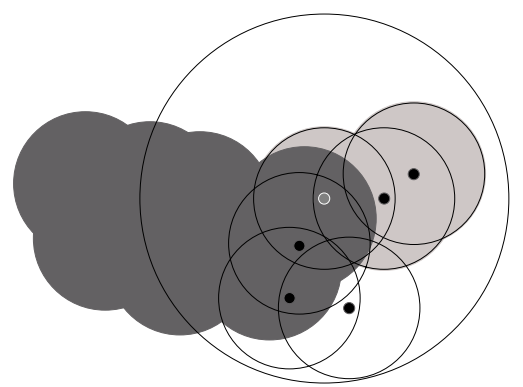

Figure 8: Conditioned (light gray), unconditioned increments (black circles) and the coupling disk (the largest circle). The radii of the disks are $r_{\text {rad }}=2 r$.

Proof. Let $N$ be an arbitrary point process. For a Boolean model with disks centered according to $N$ and radii $r$, let $W_{r}(A, N)$ denote the union of the occupied clusters intersecting set $A$ and $\tilde{W}_{r}(A, N) \doteq\left\{\mathbf{x}:\left|\mathbf{x}-\left(A \cup W_{r}(A, N)\right)\right| \leq r\right\}$ the set where the message is heard.

When $S$ grows at $t=T_{i}$, all the possible new points, except the bridge $X_{i}$, are outside $S_{T_{i-1}}$. In $S_{T_{i-1}}^{c}$ the active nodes are distributed according to a stationary Poisson point process with intensity $\lambda \pi_{\mathrm{on}}$ which we denote by $N_{i}$. Thus

$$
C\left(X_{i}\right)=\tilde{W}_{r}\left(B\left(X_{i}, r\right), N_{i}\left(S_{T_{i}-1}^{c}\right)\right) \backslash S_{T_{i}-1} .
$$

This set can be drawn without any information about nodes in $S_{T_{i}}^{c}$. Thus the increments $C\left(X_{i}\right)$ can be determined using a sequence of i.i.d. Poisson processes $N_{i}$. Moreover,

$$
C\left(X_{i}\right) \subseteq \tilde{W}_{r}\left(B\left(X_{i}, r\right), N_{i}\right)
$$

since neglecting the conditions with respect $S_{T_{i}-1}$ gives naturally a larger set (see Figure 8).

Finally, let $D_{i}=\operatorname{diam}\left(W_{r}\left(B\left(X_{i}, r\right)\right)+4 r\right.$ so that

$$
C\left(X_{i}\right) \subseteq B\left(X_{i}, D_{i}\right) .
$$

Applying Lemma 5 yields that $D_{i}$ satisfies (6).

Next we show that the process indicating when and where a bridge appears can be stochastically bounded by a Poisson process in $\mathbb{R}^{3}$. In other words, the Poisson point process includes all the bridges (plus infinitely many more), it preserves the opening order, and each bridge opens earlier than it was originally scheduled.

LEMMA 7. If the sleeping periods are either constant or exponentially distributed, then the bridge process can be coupled with a stationary Poisson point process in $\mathbb{R}^{3}$ of intensity $\lambda \pi_{\mathrm{off}} / t_{\mathrm{off}}$.

Proof. Let $\left\{X_{i}^{0}\right\}$ denote the bridges in $S_{0}$ with opening times $\left\{T_{i}^{0}\right\}$. At time $t=0$, the $X_{i}$ are distributed according to Poisson point process with intensity $\lambda \pi_{\text {off. If }} U_{i} \sim \operatorname{Uniform}\left(0, t_{\text {off }}\right)$, then points $\left(X_{i}^{0}, U_{i}\right) \in S_{0} \times\left[0, t_{\mathrm{off}}\right]$ are distributed according to a Poisson point process with intensity $\lambda \pi_{\text {off }} / t_{\text {off }}$. Thus if the $T_{i}^{0}$ are uniformly distributed then we know that they all appear according to a Poisson process on $S_{0} \times\left[0, t_{\text {off }}\right]$. On the other hand, if the $T_{i}^{0}$ are exponentially distributed, they can be coupled with the $U_{i}$ by

$$
T_{i}^{0}=-t_{\text {off }} \log \left(1-U_{i} / t_{\text {off }}\right) \geq U_{i}
$$

and thus with the Poisson point process with intensity $\lambda \pi_{\mathrm{off}} / t_{\mathrm{off}}$.

The same reasoning holds for any new set added to the cluster.
Proof. (For Proposition 5) By Lemma 6, we bound each new set added to the message cluster by disks with i.i.d. radii with cumulative distribution satisfying (6). By Lemma 7, each of the bridges originating an increase of the message set are included in a Poisson point process in $\mathbb{R}^{3}$ with intensity $\lambda \pi_{\text {off }} / t_{\text {off }}$. Thus applying Theorem 3 (shown in Appendix A) completes the proof.

\subsection{Duration of a transmission phase}

When a node receives or generates a message, it keeps transmitting until its neighbors have received the message. From an energy consumption point of view, it is important to know how long this transmitting phase will last. Assuming that the node is connected to the infinite cluster, then - in principle - it would be enough that the "optimally" located neighboring nodes receive the message. However, in order to maximize the speed at which messages travel, we define the broadcast duration as the time until all the neighboring nodes have received the message with probability $p_{b}$. Furthermore, we assume here that the emitter ignores its neighborhood and receives no feedback from the receivers. Although it would be easy to achieve better conditions by designing an appropriate protocol, we only consider this simple mechanism.

Given the transmission radius $r_{\text {rad }}=2 r$ of the sensors, there is a Poisson distributed number of sensors inside its transmission range. In a worst case scenario, we assume that all the nodes are in sleeping mode at the time when the broadcast starts. If the sleeping periods are of constant length, then the natural and $100 \%$ safe broadcasting duration is $t_{\text {off }}+\Delta$, where $\Delta$ is the propagation delay. Otherwise, we assume that the off periods of the neighbours, $T_{k}, k=$ $1, \ldots, N$, are exponentially distributed and $N \sim \operatorname{Poisson}\left(\lambda \pi r_{\text {rad }}^{2}\right)$. In order to be $p_{b}$ sure that every node receives the message, the broadcasting time $t_{b}$ has to satisfy

$$
\begin{aligned}
\mathbb{P}\left(\max \left\{T_{k}\right\}_{k=1}^{N}<t_{b}\right) & =\sum_{k=0}^{\infty} \frac{\left(\lambda \pi r_{\text {rad }}^{2}\right)^{k}}{k !} e^{-\lambda \pi r_{\text {rad }}^{2}}\left(1-e^{-t / t_{\text {off }}}\right)^{k} \\
& =\exp \left(\lambda \pi r_{\text {rad }}^{2} e^{-t / t_{\text {off }}}\right)<p_{b}
\end{aligned}
$$

Thus, if the broadcasting time $t_{b}$ satisfies

$$
t_{b} \geq t_{\mathrm{off}} \log \left(\frac{\lambda \pi r_{\mathrm{rad}}^{2}}{-\log p_{b}}\right)+\Delta,
$$

then it is $p_{b}$ sure that every node in its neighborhood gets the message.

\subsection{Simulation studies}

We have performed a series of simulations to validate the results concerning latency presented in Section 5.1. These numerical studies clearly agree with the analytical results on the linear spreading rate. Although Theorem 1 is stated only for constant transmission radii, the simulations suggest that it is also valid for random transmission radii.

All the simulations were run with intensity $\lambda=3$. The sleeping periods were drawn from an exponential distribution with mean $t_{\mathrm{off}}$. The size of the area which is reached by the message at time $t$ is measured by $\max \left|G_{t}\right|=\operatorname{argmax}\left\{|x|: x \in G_{t}\right\}$ and the spreading rate is estimated by

$$
\hat{\mu}=\frac{\mathbb{E}\left(\max \left|G_{t}\right|\right)}{t} .
$$

Simulations of the growth of the message cluster with varying period lengths $t_{\mathrm{on}}$ and $t_{\mathrm{off}}$ are shown in Figures 9 and 10. In principle, only the ratios $t_{\mathrm{on}} /\left(t_{\mathrm{off}}+t_{\mathrm{on}}\right)$ and $t_{\mathrm{off}} /\left(t_{\mathrm{off}}+t_{\mathrm{on}}\right)$ matter. By a simple time scaling argument $t_{\text {off }}$ (or $\left.t_{\text {on }}\right)$ can be taken as the time 

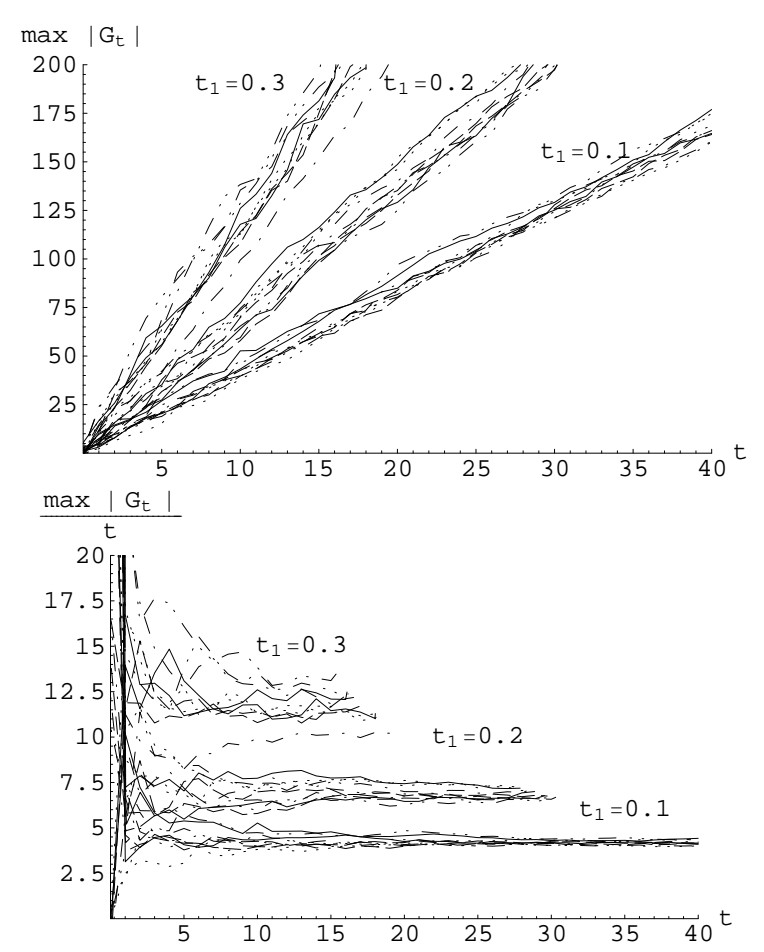

Figure 9: Maximum connection distance from the origin for different active periods. Ten independent simulations with parameters $\lambda=3, r=1, t_{\mathrm{off}}=1$, and $t_{\mathrm{on}}=0.1,0.2,0.3$
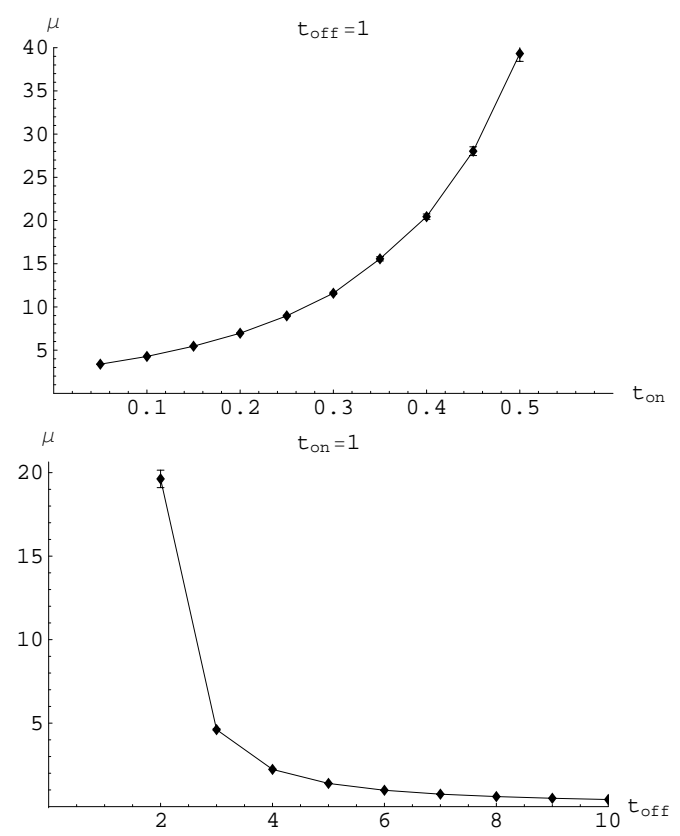

Figure 10: Estimation of growth rates. Parameters $\lambda=3, r=1$. 100 independent simulations per estimate. 
models is that in the latter longer jumps are possible. The other difference is that the mean covered area is larger in the random case, since $\mathbb{E}\left(R^{2}\right) \geq(\mathbb{E}(R))^{2}$. This naturally results in better connectivity. However we observe that the qualitative behavior is similar. Actually, most of the proofs presented in this paper can be easily extended to the random radii model, and we conjecture that all our results hold for this model.

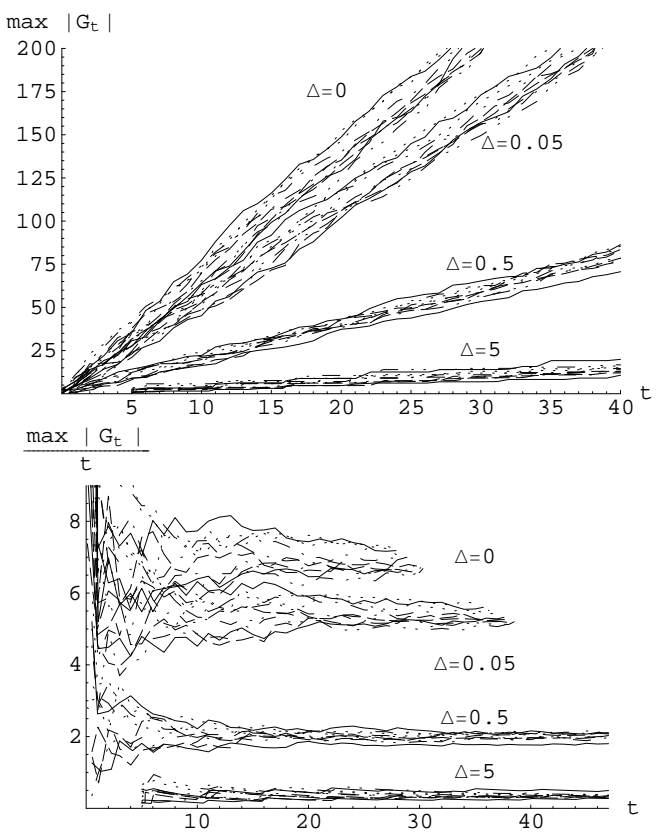

Figure 13: Model with propagation delays. Maximum connection distance from the origin. Ten independent simulations with parameters $\lambda=3, r=1, t_{\mathrm{off}}=1, t_{\mathrm{on}}=0.2$ and $\Delta=0,0.05,0.5,5$.

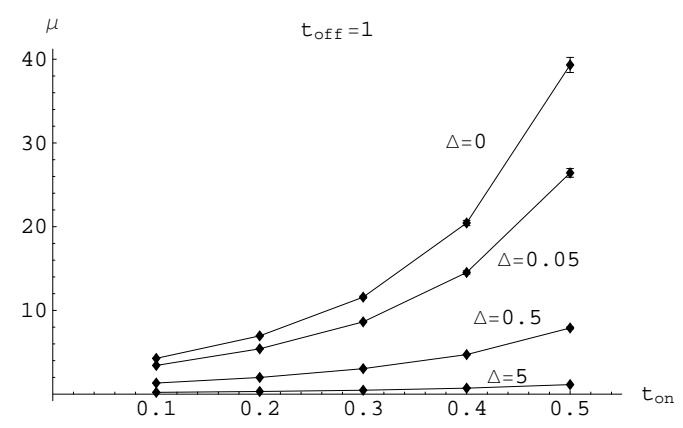

Figure 14: Model with propagation delays. Estimated growth rates. Parameters $\lambda=3, r=1, t_{\text {off }}=1$, delays $0.05,0.5$ and 5 . Estimates based on 100 independent simulations.

Figure 13 shows the impact of propagation delays on the message spreading. The asymptotic behavior is qualitatively conserved, as predicted. The quantitative impact is also tiny, if we consider the realistic case where delays are short compared to $t_{\mathrm{on}}$ and $t_{\mathrm{off}}$.

\section{CONCLUDING REMARKS}

The blinking Poisson Boolean model is suited for sensor networks where nodes switch between a sleeping and an active phase. Even though their switching on/off schedules are not coordinated at all, their positions are random, and the durations $t_{\mathrm{on}}$ and $t_{\mathrm{off}}$ are such that the number of active nodes at any particular time is so low that the network is always disconnected, we have proved that any message (alarm) generated by a sensor will reach the sink in a time proportional to the distance between the sensor and the sink. The value of the rate of this linear growth does not depend on the random locations of the nodes, but only on the parameters $\lambda$ (node density), $r$ (connectivity range), $t_{\mathrm{on}}$ and $t_{\mathrm{off}}$ (average active and sleeping durations).

In this paper, we have only considered the case where, at any particular time $t$, the network is disconnected (sub-critical phase). The other case, where the network remains in a supercritical phase at any time, is also interesting. Indeed, the latency will be much smaller than in the subcritical case, but it is not clear how to bound it. We leave this part for future study.

\section{ACKNOWLEDGMENTS}

O. Dousse's work was supported (in part) by the National Competence Center in Research on Mobile Information and Communication Systems (NCCR-MICS), a center supported by the Swiss National Science Foundation under grant number 5005-67322.

This work was done while P. Mannersalo was visiting EPFL as an ERCIM fellow. He is grateful for the generous financial support of Swiss National Science Foundation (PAER2-101377), EPFL and Academy of Finland (\#202218).

\section{REFERENCES}

[1] F. Baccelli, B. Blaszczyszyn, and F. Tournois. Spatial averages of coverage characteristics in large cdma networks. Wireless Networks, 8:569-586, 2002.

[2] J. van den Berg, R. Meester, and D.G. White. Dynamic Boolean models. Stochastic Processes and their Applications, 69:247-257, 1997.

[3] J.-H. Chang and L. Tassiulas. Energy conserving routing in wireless ad-hoc networks. In Proceedings of IEEE Infocom 2000, pages 22-31, Jerusalem, 2000.

[4] C.-F. Chiasserini and M. Garetto. Modeling the performance of wireless sensor networks. In Proceedings of IEEE Infocom 2004, 2004.

[5] M. Deijfen. Asymptotic shape in a continuum growth model. Advances in Applied Probability, 35(2):303-318, 2003.

[6] M. Deijfen, O. Häggström, and J. Bagley. A stochastic model for competing growth on $\mathbb{R}^{d}$. Markov Processes and Related Fields, 2003. To appear.

[7] O. Dousse, F. Baccelli, and P. Thiran. Impact of interferences on connectivity in ad hoc networks. IEEE/ACM Trans. Networking, accepted for publication.

[8] A. El Gamal, C. Nair, B. Prabhakar, E. Uysal-Biyikoglu, and S. Zahedi. Energy-efficient scheduling of packet transmissions over wireless networks. In Proceedings of IEEE Infocom 2002, pages 1773-1782, 2001.

[9] A. Ephremides. Energy concerns in wireless networks. IEEE Wireless Communications, 9(4):48-59, 2002.

[10] M. Franceschetti, L. Booth, J. Bruck, M. Cook, and R. Meester. Percolation in multi-hop wireless networks. IEEE Transaction on Information Theory, 2003. Submitted. A short version appeared at ISIT'03.

[11] D. Ganesan, B. Krishnamachari, A. Woo, D. Culler, D. Estrin, and S. Wicker. Complex behavior at scale: An experimental study of low-power wireless sensor networks. Technical Report UCLA/CSD-TR 02-0013, UCLA, 2002. 
[12] G. Grimmett. Percolation, volume 321 of Grundlehren der mathematischen Wissenschaften. Springer, Berlin, 2nd edition, 1999.

[13] G. Grimmett and J. Marstrand. The supercritical phase of percolation is well behaved. Proc. Royal Soc. London Ser. A, 430:439-457, 1990.

[14] O. Häggström, Y. Peres, and J. Steif. Dynamic percolation. Ann. IHP Probab. et. Statist., 33:497-528, 1997.

[15] B. Krishanamachari, D. Estrin, and S. Wicker. The impact of data aggregation in wireless sensor networks. In Proc. of International Workshop of Distributed Event Based Systems (DEBS), Vienna, Austria, 2002.

[16] T. Liggett. An improved subadditive ergodic theorem. The Annals of Probability, 13(4):1279-1285, 1985.

[17] B. Liu and D. Towsley. On the coverage and detectability of wireless sensor networks. In Proceedings of WiOpt'03. Modeling and Optimization in Mobile, Ad Hoc and Wireless Networks, Sophia Antipolis, France, 2003.

[18] R. Meester and R. Roy. Continuum percolation, volume 119 of Cambridge tracts in mathematics. Cambridge University Press, 1996.

[19] Y. Peres and J. Steif. The number of infinite clusters in dynamical percolation. Probab. Th. Rel. Fields, 111:141-165, 1998.

[20] B. Prabhakar, E. Uysal-Biyikoglu, and A. El Gamal. Energy-efficient transmission over a wireless link via lazy packet scheduling. In Proceedings of IEEE Infocom 2001, pages 386-394, 2001

[21] J. Quintanilla, S. Torquato, and R.M. Ziff. Efficient measurement of the percolation threshold for fully penetrable discs. J. Phys. A, 33:399-407, 2000.

[22] S. Singh, M Woo, and C.S. Raghavendra. Power-aware routing in mobile ad hoc networks. In Proc. of ACM Mobicom 1998, pages 181-190, 1998.

[23] A. Sinha and A. Chandrakasan. Dynamic power management in wireless sensor networks. IEEE Design and Test of Computers Magazine, 18(2):62-74, 2001.

[24] D. Stoyan, W.S. Kendall, and J. Mecke. Stochastic geometry and its applications. Wiley, Chichester, 2nd edition, 1995.

[25] T. Vicsek and J. Kertész. Monte Carlo renormalisation group approach to percolation on a continuum: Test of universality. J. Phys. A, 14(L31), 1981.

[26] Y. Wei, J. Heidemann, and D. Estrin. An energy-efficient MAC protocol for wireless sensor networks. In Proc. IEEE Infocom, New York, June 2002.

[27] W. Ye and J. Heidemann. Medium access control in wireless sensor networks. Technical Report ISI-TR-580, USC, 2003.

[28] Y. Yu, B. Krishnamachari, and V. Prasanna. Energy-latency tradeoffs for data gathering in wireless sensor networks. In Proceedings of IEEE Infocom 2004, 2004.

\section{APPENDIX}

\section{A. CONTINUUM GROWTH MODEL}

Continuum growth model is a model for a spreading infection. Assuming that an infection has spread to set $S_{t}$ at time $t$, the time until the next outburst occurs somewhere in $S_{t}$ is exponentially distributed with parameter $\lambda v_{d}\left(S_{t}\right)$ and the location of the outburst is uniformly distributed over $S_{t}$. Outburst are assumed to be balls with i.i.d. random radii. This model was first studied by Deijfen [5] (see also Deijfen, Häggström and Bagley [6]).
Formally, Continuum growth model is defined as follows. We start with a stationary Poisson point process $N=\left(X_{k}, T_{k}\right)_{k} \in \mathbb{R}^{d+1}$ with $X_{k} \in \mathbb{R}^{d}$. The Poisson process has intensity $\lambda$ and the points laying inside a set $G$ are denoted by $N(G)=G \cap N$. For each $X_{k}$ we attach a ball $B\left(X_{k}, D_{k}\right)=B_{k} \subset \mathbb{R}^{d}$ which is centered at $X_{k}$ and has a random radius $D_{k}$. Assume the radii $D_{k}$ i.i.d. with a common cumulative distribution function $F$.

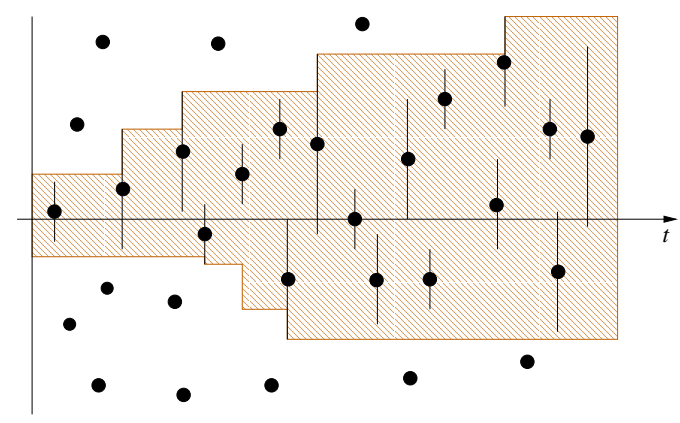

Figure 15: Continuum growth model in $\mathbb{R}^{2} . S_{t}$ is shown by the shaded area.

Let us enumerate points of $N$ as follows. Let $X_{0}=0, T_{0}=0$, $D_{0}=\gamma$, and $S_{0}=B(0, \gamma)=B_{0}$. Given $\left(X_{k}, T_{k}, D_{k}\right), k=0, \ldots, n$,

$$
T_{n+1}=\inf _{k}\left\{T_{k}: T_{k}>T_{n},\left(X_{k}, T_{k}\right) \in N\left(\bigcup_{i=0}^{n} B_{i} \times \mathbb{R}\right)\right\} .
$$

For each $T_{n}$ there is (a.s. unique) $X_{n}$ and a random radius $D_{n}$.

Continuum growth process $S_{t} \subset \mathbb{R}^{d}$ is a Markov process which is constructed from the sequence of the $B_{i}$ :

$$
S_{t}=\bigcup_{i=0}^{n(t)} B_{i}
$$

where $n=\inf _{k}\left\{k: T_{k} \geq t\right\}$. Figure 15 shows an example where $S_{t} \subset \mathbb{R}$.

For our purposes, the main property of Continuum growth model is that the size of the infected area grows asymptotically linearly.

Theorem 3. [6] Fix $d \geq 1$ and consider the $d$-dimensional continuum growth model with rate $\lambda$. Assume that

$$
\int_{0}^{\infty} e^{-\varphi r} \mathrm{~d} F(r)<\infty
$$

for some $\varphi<0$ and let $S_{0} \subset \mathbb{R}^{d}$ be arbitrary but bounded with strictly positive Lebesgue measure. Then there exists a real number $\mu>0$ such that for any $\varepsilon \in\left(0, \lambda \mu^{-1}\right)$, almost surely

$$
(1-\varepsilon) B\left(0, \lambda \mu^{-1}\right) \subseteq \frac{S_{t}}{t} \subseteq(1+\varepsilon) B\left(0, \lambda \mu^{-1}\right)
$$

for all sufficiently large t. Moreover, the time constant $\mu$ is given by

$$
\mu=\lim _{n \rightarrow \infty} \frac{\mathbb{E}(\tilde{T}(\mathbf{n}))}{n}=\lim _{n \rightarrow \infty} \frac{\tilde{T}(\mathbf{n})}{n},
$$

where $\mathbf{n}=(n, 0, \ldots, 0)$ and $\tilde{T}(x)=\inf \left\{t: B(x, \gamma) \subseteq S_{t}\right\}$. 